\title{
Tomography using annular dark field imaging in TEM
}

\author{
S. Bals, ${ }^{*}$ C. Kisielowski, ${ }^{* *}$ M. Croitoru, ${ }^{*}$ and G. Van Tendeloo* \\ * EMAT-University of Antwerp, Groenenborgerlaan 171, Antwerp B-2020, Belgium \\ ** National Center for Electron Microscopy, Lawrence Berkeley National Laboratory, One \\ Cyclotron Road, Berkeley CA 94720, USA
}

The use of bright field TEM for tomography in physical sciences is often hampered by Bragg contributions. In Figure 1 it is illustrated that the morphology of Pt nanoparticles (5-7 nm) is well reconstructed, but artificial cavities are observed inside the $3 \mathrm{D}$ reconstructions of the particles. The different HREM projections in a tilt series of a Pt nanoparticle are simulated using multislice calculations. Using these images as an input for weighted back projection, artificial cavities are again found in the $3 \mathrm{D}$ reconstruction. This is not the case when we use the simulated phase of the exit wave as an input for $3 \mathrm{D}$ reconstruction. This allows us to conclude that strong scattering and channeling effects are possible reasons for the formation of these artefacts.

An experimental method to avoid Bragg contributions is to combine electron tomography with annular dark field TEM (ADF-TEM) [1-3]. In this technique, the central beam and all electrons scattered up to a certain semi-angle are excluded from imaging by an annular objective aperture. In this manner, the traditional high-resolution TEM image contrast (linear interference between the central beam and diffracted beams) is suppressed and a mass-thickness contrast is generated that depends exponentially on sample thickness. This means that ADF-TEM fulfills the projection requirement and that the technique can be used for tomography.

Using this approach, we have successfully obtained a 3D reconstruction of CdTe tetrapods as shown in Figure 2. It is found that ADF-TEM is a chemically sensitive technique and a heavier material will appear with higher intensity compared to a lighter material. This explains the indentation observed in one of the branches of the 3D reconstruction of the tetrapod. This area corresponds to the CdSe seed used during growth of the CdTe tetrapods [4]. The indentation does not correspond to a thinner part in one of the branches since the bright field TEM tomogram presented in Figure 3 shows that all branches have equal thickness.

An additional advantage of using ADF-TEM for tomography is that the images require exposure times of only 3-5 seconds, which means that the acquisition time of a tomographic tilt series is remarkably reduced compared to e.g. HAADF-STEM or EFTEM where longer scanning times or multiple images for every tilt angle are required. Also scanning noise is avoided using the approach proposed in this work [5].

References

[1] K. Heinemann and H. Poppa. Appl. Phys. Lett. 20 (1972) 122

[2] S. Bals et al. Solid State Communications 130 (2004) 675

[3] S. Bals et al. submitted to Ultramicroscopy

[4] L. Manna et al. Nature Materials 2 (2003) 382 
[5] This work was supported by the Director, Office of Science, Office of Basic Energy Sciences, of the US Department of Energy under Contract No. DE-AC03-76SF00098. S. Bals is grateful to the Fund of Scientific Research-Flanders.

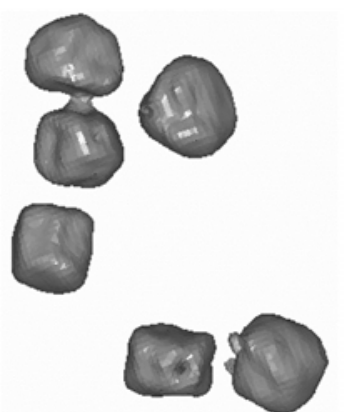

a)

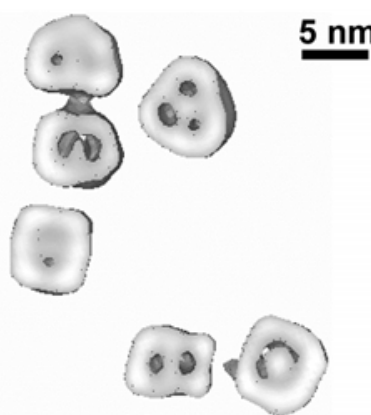

b)

FIG. 1. a) 3D iso-volume visualization of Pt nano particles b) inside the particles, artificial cavities are observed

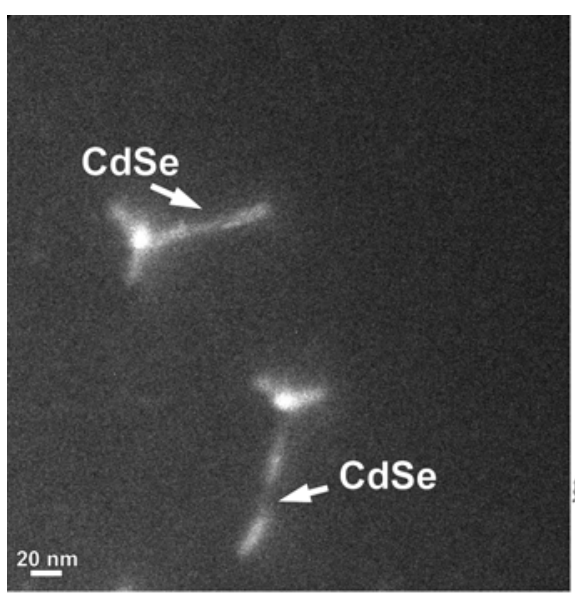

a)

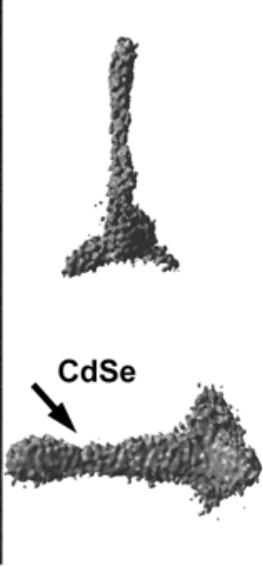

b)

FIG. 2. a) Individual ADF-TEM projection from the tomographic tilt series, the CdSe seed is imaged with less intensity b) the ADF tomogram of the CdTe tetrapod shows an indentation in one of the tetrapods branches corresponding to the CdSe seed.

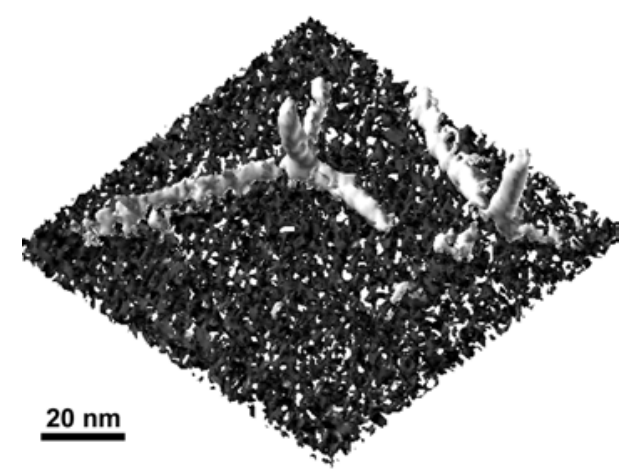

FIG. 3. 3D iso-surface visualization of a bright field TEM tomogram showing 2 CdTe tetrapods on a carbon foil. 